\title{
Increased Hematocrit During Sodium-Glucose Cotransporter 2 Inhibitor Therapy Indicates Recovery of Tubulointerstitial Function in Diabetic Kidneys
}

\author{
Motoaki Sano $^{\text {a, c }}$, Makoto Takei ${ }^{a}$, Yasuyuki Shiraishi ${ }^{a}$, Yoshihiko Suzuki ${ }^{b}$
}

\begin{abstract}
Sodium-glucose cotransporter 2 (SGLT2) inhibitors have been attracting attention for cardiovascular as well as antidiabetic effects since the results of the Empagliflozin Cardiovascular Outcome Event Trial in Type 2 Diabetes Mellitus Patients (EMPA-REG OUTCOME Trial) were reported. The hematocrit increases during treatment with SGLT2 inhibitors, which have a diuretic effect but do not cause sufficient hemoconcentration to increase the risk of cerebral infarction. Elevation of the hematocrit during SGLT2 inhibitor therapy is presumed to involve enhancement of erythropoiesis in addition to hemoconcentration. In patients with diabetes, the erythropoietin level increases after initiation of treatment with the SGLT2 inhibitor dapagliflozin and reaches a plateau in 2 - 4 weeks. The reticulocyte count increases simultaneously, followed by elevation of hemoglobin and hematocrit. In patients with diabetes, the proximal tubules are overtaxed by excessive glucose reabsorption and the increased oxygen requirement causes tubulointerstitial hypoxia. Consequently, erythropoietin production is impaired because "neural crest-derived" fibroblasts surrounding the damaged renal tubules undergo transformation into dysfunctional fibroblasts. SGLT2 inhibitors reduce the workload of the proximal tubules and improve tubulointerstitial hypoxia, allowing fibroblasts to resume normal erythropoietin production. These drugs represent a new class of diuretics that have a renoprotective effect by improving tubulointerstitial hypoxia, which is the final common pathway to end-stage renal disease. In patients with diabetes, elevation of hematocrit may be a surrogate marker for recovery from reversible tubulointerstitial injury.
\end{abstract}

Keywords: Type 2 diabetes; SGLT2 inhibitor; Antidiabetic agent; Cardiovascular medication; Reversible tubulointerstitial injury; Hematocrit; Renoprotective effect

Manuscript accepted for publication September 29, 2016

${ }^{\text {aDepartment }}$ of Cardiology, Keio University School of Medicine, Tokyo, Japan

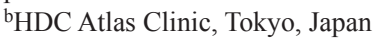

${ }^{\mathrm{c} C}$ Corresponding Author: Motoaki Sano, Department of Cardiology, Keio University School of Medicine, 35 Shinanomachi Shinjuku-ku, Tokyo 160-8582, Japan.Email: msano@a8.keio.jp

doi: http://dx.doi.org/10.14740/jocmr2760w

\section{Introduction}

The Empagliflozin Cardiovascular Outcome Event Trial in Type 2 Diabetes Mellitus Patients (EMPA-REG OUTCOME) [1] demonstrated that treatment with empagliflozin, a sodiumglucose cotransporter 2 (SGLT2) inhibitor, decreased cardiovascular death by $38 \%$ in patients with type 2 diabetes at high risk of cardiovascular events and also reduced admission to hospital for heart failure by $35 \%$. Before the results of this study were reported, pooled analyses of clinical studies of canagliflozin [2] and dapagliflozin [3, 4] showed that dapagliflozin treatment also significantly reduced hospitalization for cardiac failure (hazard ratio: $0.57,95 \%$ confidence interval (CI): $0.34-0.95$ ) and myocardial infarction (hazard ratio: 0.36, $95 \%$ CI: 0.16 - 0.84) compared with placebo. A meta-analysis of SGLT2 inhibitor therapy in more than 30,000 patients published in 2016 [5] revealed that the relative risk of three-point major adverse cardiac events (MACEs) was reduced to 0.84 (95\% CI: 0.75 - 0.95), confirming the efficacy of four SGLT2 inhibitors (dapagliflozin, canagliflozin, empagliflozin, and ipragliflozin) for significantly reducing cardiovascular events. Thus, the cardiovascular effects of SGLT2 inhibitors have been attracting attention.

The hematocrit increases soon after initiation of SGLT2 inhibitor therapy, and remains elevated as long as SGLT2 inhibitor administration continues [1]. Elevation of the hematocrit has generally been interpreted as indicating hemoconcentration due to the diuretic effect of SGLT2 inhibitors, although it has been reported that the risk of cerebral infarction is not increased [1-5]. Nevertheless, many clinicians consider that an increase in the hematocrit in patients on SGLT2 inhibitor therapy is associated with an elevated risk of cerebral infarction, and discontinue these drugs in patients with excessive hematocrit elevation.

\section{Does Elevation of the Hematocrit in Patients on SGLT2 Inhibitor Therapy Reflect Hemoconcen- tration Alone?}

Diuretics, particularly loop diuretics, are required for treating pulmonary congestion and edema in patients with heart failure complicated by fluid retention. On the other hand, overuse of 


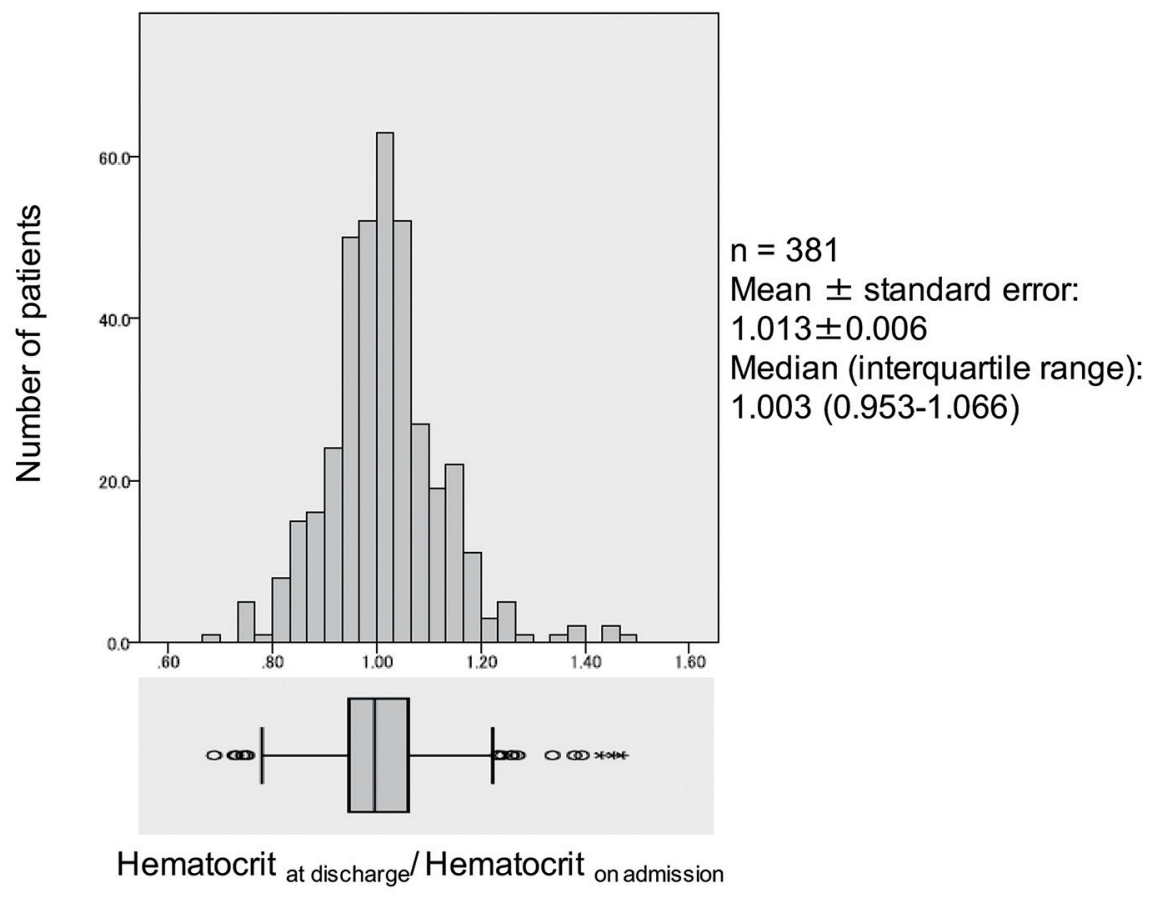

Figure 1. Distribution of in-hospital changes in hematocrit.

loop diuretics causes deterioration of renal function in patients with heart failure and is associated with a worse prognosis [6], and these medications should be restricted to the minimum dosage required. Therefore, cardiovascular physicians determine the optimum dose of a loop diuretic by paying attention to blood urea nitrogen and creatinine levels or the blood urea nitrogen/creatinine ratio, in order to suppress activation of neurohumoral factors, maintain adequate circulating blood volume, and avoid deterioration of renal function. It should be noted that these physicians do not use changes in the hematocrit as an index of hemoconcentration during diuretic therapy.

The hematocrit at hospital admission and discharge was compared in 381 patients (251 men) with a mean age of 69.2 years who were admitted to Keio University Hospital because of heart failure between January 2007 and August 2014 (Fig. 1). Loop diuretics were administered to most of the patients to alleviate symptoms such as pulmonary congestion and edema. Data on the hospital discharge/admission hematocrit ratio showed an approximately normal distribution, with a mean value of $1.013 \pm 0.006$ and median of 1.003 (first quartile: 0.953, third quartile: 1.066). In some patients, the hematocrit increased after starting treatment with loop diuretics, whereas it decreased in other patients. Accordingly, the change in hematocrit after initiation of diuretic therapy is not a reliable index for estimating the increase or decrease in total body fluid and effective circulating blood volume in patients with heart failure. Similar results were obtained by investigation of the changes in hematocrit during hospitalization in 1,684 patients from the placebo group of the Efficacy of Vasopressin Antagonism in Heart Failure Outcome Study with Tolvaptan (EVEREST) study who were hospitalized for heart failure with an ejection fraction $\leq 40 \%$ [7]. Thus, it seems that changes in the hematocrit during diuretic therapy are largely dependent on factors other than hemoconcentration.

The mechanism underlying elevation of the hematocrit during SGLT2 inhibitor therapy is presumed to involve enhancement of erythropoiesis. The kidney produces erythropoietin, which is a hormone required for erythropoiesis. Renal fibroblasts originate from neural crest cells that migrate to the kidney during development. These "neural crest-derived" fibroblasts produce erythropoietin when the renal tubules are normal, but undergo transformation into dysfunctional fibroblasts with decreased erythropoietin production after tubular injury, resulting in nephrogenic anemia and kidney fibrosis. Fibroblast transformation is reversible when renal tubular injury is mild, and these cells can revert to healthy fibroblasts if the kidney responds to appropriate treatment. However, advanced renal tubular injury may lead to irreversible transformation to fibroblasts [8].

\section{Possible Mechanism by Which SGLT2 Inhibitors Increase Erythropoietin Production}

In patients with diabetes, administration of dapagliflozin causes the erythropoietin concentration to increase until it peaks after 2 - 4 weeks of treatment. The reticulocyte count also increases transiently at the same time, followed by elevation of hemoglobin and hematocrit [9]. Accordingly, we propose the following hypothesis concerning the renoprotective effect of SGLT2 inhibitors (Fig. 2). In patients with diabetes, the proximal tubules are overtaxed due to excessive glucose reabsorption, leading to dysfunction of adjacent "neural crest-derived" fibroblasts and reduced erythropoietin production. SGLT2 inhibitors reduce the workload of the proximal tubules, allowing 


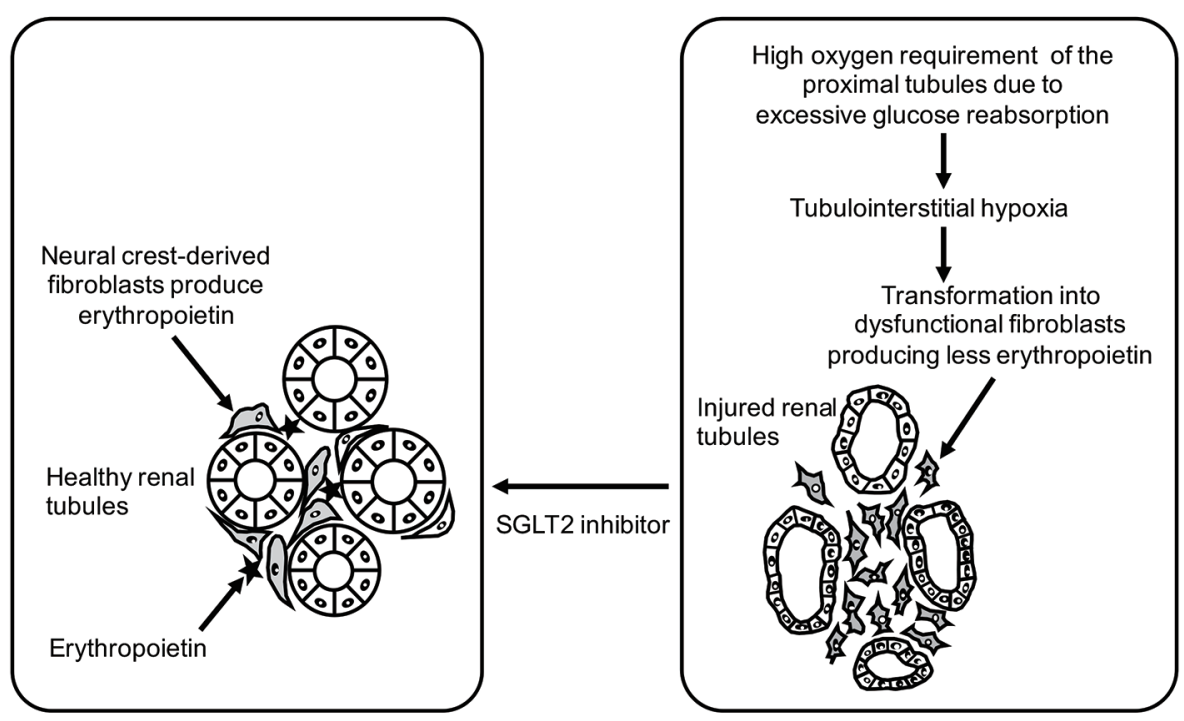

Figure 2. Functional recovery of tubulointerstitial fibroblasts with SGLT2 inhibitor therapy (reverse remodeling). SGLT2 inhibitor therapy suppresses oxygen consumption by the proximal tubules and improves tubulointerstitial hypoxia.

restoration of tubulointerstitial function and increased production of erythropoietin by "neural crest-derived" fibroblasts.

Findings obtained in animal models have provided support for this hypothesis. Recent research has suggested that tubulointerstitial hypoxia is the final common pathway leading to end-stage renal disease [10]. Oxygen consumption by the proximal tubules is higher in patients with diabetes than healthy persons because of an increased workload due to excessive glucose reabsorption and the tissue partial pressure of oxygen in the renal cortex is accordingly lower, while renal cortical hypoxia is improved by administration of phlorizin, the prototype SGLT2 inhibitor [11]. It was also reported that dapagliflozin reduced oxidative stress due to excessive oxygen consumption in a mouse model of diabetic nephropathy [12]. SGLT2 inhibitor treatment is associated with elevation of circulating ketone bodies ( $\beta$-hydroxybutyrate), which inhibit class I histone deacetylase activity [13]. Elevation of $\beta$-hydroxybutyrate increases the acetylation of renal histones $\mathrm{H} 3 \mathrm{~K} 9$ and H3K14, leading to induction of genes promoting resistance to oxidative stress. Taken together, the increase in hematocrit during SGLT2 inhibitor therapy may indicate improvement of hypoxia and oxidative stress in the tubulointerstitial region of the renal cortex, as well as recovery of erythropoietin production by "neural crest-derived" fibroblasts.

Based on these observations, it seems reasonable to assume that an increase in hematocrit in patients taking SGLT2 inhibitors is associated with reverse renal remodeling. Subanalysis of the EMPA-REG OUTCOME Trial suggested that elevation of hematocrit during empagliflozin therapy had the strongest association with reduction of cardiovascular death [14]. Furthermore, EMPA-REG Renal OUTCOME Trial showed substantial improvement of renal outcomes by empagliflozin, including a 39\% decrease in the progression of nephropathy, a $44 \%$ decrease in doubling of serum creatinine, and a $55 \%$ decrease in initiation of renal replacement therapy [15]. Thus, SGLT2 inhibitors can be regarded as a new class of diuretic with a renoprotective effect related to amelioration of tubulointerstitial hypoxia and oxidative stress, the final common pathway to end-stage renal disease. SGLT2 inhibitors also reduce hospitalization for heart failure and cardiovascular death by improving cardio-renal syndrome. An increase in the hematocrit during SGLT2 inhibitor therapy may be a surrogate marker for the recovery of tubulointerstitial function. Loop diuretics achieve sufficient hemoconcentration by their potent diuretic effect, but this might mask hematocrit fluctuations secondary to reduced erythropoietin production because of tubulointerstitial injury. The hematocrit may even be reduced in patients on treatment with loop diuretics due to potential tubulointerstitial toxicity.

Because SGLT2 inhibitors should be regarded as cardiovascular medications rather than simply being viewed as glucose-lowering agents, suddenly discontinuing SGLT2 inhibitor therapy may not be without risk. In fact, fatal or non-fatal stroke has often been reported from 30 days or later after discontinuation of SGLT2 inhibitor treatment [1]. Accordingly, care should be exercised when discontinuing SGLT2 inhibitor therapy.

\section{Conclusion}

SGLT2 inhibitors represent a new class of diuretic with a renoprotective effect based on improvement of tubulointerstitial hypoxia and oxidative stress, which is the final common pathway leading to end-stage renal disease. An increase in hematocrit unaccompanied by elevation of the blood urea nitrogen/ creatinine ratio during SGLT2 inhibitor therapy is not associated with a higher risk of cerebral infarction. In fact, it may 
represent a surrogate marker of recovery from tubulointerstitial dysfunction in diabetic kidneys.

\section{References}

1. Zinman B, Wanner C, Lachin JM, Fitchett D, Bluhmki E, Hantel S, Mattheus M, et al. Empagliflozin, Cardiovascular Outcomes, and Mortality in Type 2 Diabetes. N Engl J Med. 2015;373(22):2117-2128.

2. US Food and Drug Administration. Canagliflozin as an adjunctive treatment to diet and exercise alone or coadministered with other antihyperglycemic agents to improve glycemic control in adults with type 2 diabetes mellitus. Silver Spring; Food and Drug Administration, 2013. http://www.fda.gov/downloads/AdvisoryCommittees/CommitteesMeetingMaterials/Drugs/EndocrinologicandMetabolicDrugsAdvisoryCommittee/UCM334551. pdf. (accessed Aug 30, 2016).

3. US Food and Drug Administration. FDA Briefing Document. NDA 202293. Dapagliflozin oral tablets, 5 and 10 MG. Silver Spring; Food and Drug Administration, 2013. http://www.fda.gov/downloads/AdvisoryCommittees/ CommitteesMeetingMaterials/Drugs/EndocrinologicandMetabolicDrugsAdvisoryCommittee/UCM378076.pdf. (accessed Aug 30, 2016).

4. Gilbert RE, Krum H. Heart failure in diabetes: effects of anti-hyperglycaemic drug therapy. Lancet. 2015;385(9982):2107-2117.

5. Wu JH, Foote C, Blomster J, Toyama T, Perkovic V, Sundstrom J, Neal B. Effects of sodium-glucose cotransporter-2 inhibitors on cardiovascular events, death, and major safety outcomes in adults with type 2 diabetes: a systematic review and meta-analysis. Lancet Diabetes Endocrinol. 2016;4(5):411-419.

6. Hasselblad V, Gattis Stough W, Shah MR, Lokhnygina Y, O'Connor CM, Califf RM, Adams KF, Jr. Relation between dose of loop diuretics and outcomes in a heart failure population: results of the ESCAPE trial. Eur J Heart Fail. 2007;9(10):1064-1069.
7. Greene SJ, Gheorghiade M, Vaduganathan M, Ambrosy AP, Mentz RJ, Subacius H, Maggioni AP, et al. Haemoconcentration, renal function, and post-discharge outcomes among patients hospitalized for heart failure with reduced ejection fraction: insights from the EVEREST trial. Eur J Heart Fail. 2013;15(12):1401-1411.

8. Asada N, Takase M, Nakamura J, Oguchi A, Asada M, Suzuki N, Yamamura K, et al. Dysfunction of fibroblasts of extrarenal origin underlies renal fibrosis and renal anemia in mice. J Clin Invest. 2011;121(10):3981-3990.

9. Lambers Heerspink HJ, de Zeeuw D, Wie L, Leslie B, List J. Dapagliflozin a glucose-regulating drug with diuretic properties in subjects with type 2 diabetes. Diabetes Obes Metab. 2013;15(9):853-862.

10. Nangaku M. Chronic hypoxia and tubulointerstitial injury: a final common pathway to end-stage renal failure. J Am Soc Nephrol. 2006;17(1):17-25.

11. O'Neill J, Fasching A, Pihl L, Patinha D, Franzen S, Palm F. Acute SGLT inhibition normalizes O2 tension in the renal cortex but causes hypoxia in the renal medulla in anaesthetized control and diabetic rats. Am J Physiol Renal Physiol. 2015;309(3):F227-234.

12. Terami N, Ogawa D, Tachibana H, Hatanaka T, Wada J, Nakatsuka A, Eguchi J, et al. Long-term treatment with the sodium glucose cotransporter 2 inhibitor, dapagliflozin, ameliorates glucose homeostasis and diabetic nephropathy in db/db mice. PLoS One. 2014;9(6):e100777.

13. Shimazu T, Hirschey MD, Newman J, He W, Shirakawa K, Le Moan N, Grueter CA, et al. Suppression of oxidative stress by beta-hydroxybutyrate, an endogenous histone deacetylase inhibitor. Science. 2013;339(6116):211214.

14. Zinman B, McGuire DK, Wanner C, Lachin JM, Inzucchi SE, Herman WH. Update from EMPA-REG OUTCOME Trial. 3-CT-SY29 at the American Diabetes Association Annual Scientific Meeting New Orleans, June 2016.

15. Wanner C, Inzucchi SE, Lachin JM, Fitchett D, von Eynatten M, Mattheus M, Johansen OE, et al. Empagliflozin and Progression of Kidney Disease in Type 2 Diabetes. $\mathrm{N}$ Engl J Med. 2016;375(4):323-334. 